\title{
ON ALGEBRAS OF FINITE REPRESENTATION TYPE
}

BY

\author{
SPENCER E. DICKSON( $\left.{ }^{1}\right)$
}

Introduction. Since D. G. Higman proved that bounded representation type and finite representation type are equivalent for group algebras at prime characteristic, there has been a renewed interest in the Brauer-Thrall conjecture that bounded representation type implies finite representation type for arbitrary algebras. The main purpose of this paper is to present a new approach to this conjecture by showing the relevance (when the base field is algebraically closed) of questions concerning the structure of indecomposable modules of certain special types, namely, the stable (every maximal submodule is indecomposable), the costable (having the dual property), and the stable-costable (having both properties) indecomposable modules. The main tools are the Sandwich Lemma (1.2) which is proved using an old observation of É. Goursat, an observation of A. Heller, C. W. Curtis, and D. Zelinsky concerning quasifrobenius (QF) rings (Proposition 2.1), and a general interlacing technique similar to methods used by Jans, Tachikawa, and Colby for building up large indecomposable modules of finite length which has validity in any abelian category (Theorem 3.1).

In $\S 1$ we give an alternate approach to recent results of C. W. Curtis and J. P. Jans [4] which give sufficient conditions regarding the structure of all indecomposables in order that the algebra will have finite module type. We give sufficient conditions on the structure of the stable (resp. costable, stable-costable) indecomposables in order that $A$ will have at most finitely many isomorphism classes of modules of any finite composition length. We abbreviate this by saying that $A$ has $\omega$-finite module type, where $\omega$ denotes the first infinite ordinal.

In $\$ 2$ we prove several properties of indecomposables over quasifrobenius algebras. Curtis and Jans in [4] showed that if $A$ is any algebra over an algebraically closed field such that each indecomposable module has square-free socle (i.e., it contains no submodule of the forms $S \oplus S$ for simple $S$ ), then $A$ has finite module type. This condition also implies that if $\varphi$ is a nilpotent endomorphism of an indecomposable module $M$, then $\varphi$ kills the entire socle of $M$. We say that such a module has large kernels, and if a ring $A$ has this property for all its indecomposables of finite length, we say that $A$ has large kernels. We suspect that the hypothesis

Received by the editors August 30, 1967.

( $\left.{ }^{1}\right)$ The author gratefully acknowledges support by a Postdoctoral Research Associateship awarded by the Office of Naval Research under Contract No. N00014-66-C0293, NR 043346 at the University of Oregon, while on leave from the University of Nebraska during the year 1966-1967. Reproduction in whole or in part is permitted for any purpose of the United States Government. 
of large kernels is much weaker than the hypothesis of square-free socles for QF algebras over an algebraically closed field (indeed, in an earlier version of this paper we thought we had proved that any QF ring had large kernels-we still have no counterexample at the time of this writing $)\left({ }^{2}\right)$. Under the hypothesis of large kernels, we show that a fairly large class of the indecomposables over a $\mathrm{QF}$ algebra $A$ must either have square-free socles, or $A$ has infinitely many nonisomorphic indecomposables of the same composition length (Theorem 2.5).

In $\$ 3$ we prove (Theorem 3.1) that if $M$ is an indecomposable module of finite composition length over any ring $A$ and $C, C^{\prime}$ are isomorphic submodules of $M$ satisfying $C R \cap C^{\prime} R=\{0\}=C \mathscr{N}=C^{\prime} \mathscr{N}$ (where $R=\operatorname{End}_{A}(M)$ and $\mathscr{N}=\operatorname{Rad} R$ ) and $\operatorname{Ext}_{A}{ }^{1}(M, C)=0$, then $A$ has indecomposable left modules of arbitrarily large (finite) composition length. We apply this result to show (Theorem 3.6) that if $A$ is a QF algebra of bounded module type with large kernels over an algebraically closed field such that each stable-costable indecomposable module $E$ has either a maximal submodule $M$ with $\operatorname{Hom}_{A}(M, E / M)=0$ or has a factor module $E / S$ with $S$ simple and $\operatorname{Hom}_{A}(S, E / S)=0$ then $A$ has finite module type. Finally an affirmative answer is obtained to a question of Curtis and Jans in the case that $A$ is a QF algebra with large kernels over an algebraically closed field having no indecomposables of length two with isomorphic composition factors (Corollary 3.7).

It is a pleasure to acknowledge some helpful correspondence and conversations with Gerald Janusz, and I am especially grateful to Charles Curtis for several valuable suggestions and for making some unpublished research data available to me.

1. Stable, costable, and stable-costable indecomposables. Unless otherwise specified, $A$ will denote a ring with unit, associative, and usually having minimum condition (on left ideals). When $A$ is an algebra, it will be finite-dimensional over an algebraically closed base field $K$. Modules will be unitary left $A$-modules, unless otherwise specified.

As we will sometimes be concerned with more general rings than algebras, we shall use the following terminology instead of referring to representations (see [4]). The ring $A$ is said to have finite module type (for left modules), if there are at most finitely many isomorphism classes of indecomposable left modules of finite (composition) length. We say $A$ has bounded module type (for left modules), if there is a positive integer $n$ such that every indecomposable left module of finite length has length at most $n$. We say $A$ has $\omega$-finite module type (for left modules), if for any positive integer $n$, there are at most finitely many indecomposables of length $n$. We do not know if "right" can be interchanged with "left" in any of these concepts if $\boldsymbol{A}$ is a general ring, but of course it is true for finite-dimensional algebras.

( $\left.{ }^{2}\right)$ Added in proof. J. P. Jans has kindly communicated to me an example of a quasifrobenius algebra not having large kernels. It has unbounded module type. 
Let $A$ have minimum condition. If $E$ is an indecomposable module having the property that every maximal submodule is indecomposable, we say that $E$ is stable. If $E$ has the property that every factor module by a simple submodule is indecomposable, we say $E$ is costable, and say that $E$ is stable-costable if both properties hold. Given a nonsplit exact sequence $0 \rightarrow M \rightarrow E \rightarrow S \rightarrow 0$ with $S$ simple, we say that $E$ is a proper simple extension of $M$. If the arrows are reversed, we say $E$ is a proper simple coextension of $M$. Reasons for our choice of the term "stable" will be found in the following result, where $M$ denotes a left module.

Proposition 1.1. (i) If $M$ is not injective, then $M$ has a proper simple extension. (ii) If $M$ is finitely generated and also indecomposable, then $M$ is stable if and only if each proper simple extension is indecomposable.

Proof. To prove (i), assume to the contrary that $\operatorname{Ext}_{A}{ }^{1}(S, M)=0$ for all simple $S$. Let $F$ be an arbitrary module. We show that $\operatorname{Ext}_{A}{ }^{1}(F, M)=0$ by induction on the least integer $k$ such that $N^{k} F=0$, where $N$ is the radical of $A$. First suppose that $N F=0$. Then $F=\sum_{\alpha \in I} S_{\alpha}$, where $S_{\alpha}$ is simple for each $\alpha \in I$, and the sum is direct. But then

$$
\operatorname{Ext}_{A}^{1}(F, M) \approx \prod_{\alpha \in I} \operatorname{Ext}_{A}^{1}\left(S_{\alpha}, M\right)=0 .
$$

Then application of $\operatorname{Ext}_{A}{ }^{1}(, M)$ to the sequence

$$
0 \rightarrow N^{k-1} F \rightarrow F \rightarrow F / N^{k-1} F \rightarrow 0
$$

shows that $M$ is injective.

For (ii), let $M$ be a finitely generated noninjective indecomposable module and suppose that $M$ has a decomposable maximal submodule $M_{1} \oplus M_{2}$ with $M / M_{1}$ $\oplus M_{2} \approx S$ simple. We obtain exact sequences

$$
\begin{aligned}
& 0 \rightarrow M_{1} \rightarrow M / M_{2} \rightarrow S \rightarrow 0, \\
& 0 \rightarrow M_{2} \rightarrow M / M_{1} \rightarrow S \rightarrow 0
\end{aligned}
$$

neither of which is split exact. To see this, use a length argument and the indecomposability of $M$ to get that $M_{2}$ is maximal in $M$ with respect to $M_{1} \cap M_{2}=0$. Hence $M / M_{2}$ is an essential extension of $M_{1} \approx M_{1} \oplus M_{2} / M_{2}$ so that (2) does not split. Similarly (1) does not split. Hence it follows that $M / M_{1} \oplus M / M_{2}$ is an essential extension of the copy of $M_{1} \oplus M_{2}$ contained in the image of $M$ under the diagonal monomorphism $M \rightarrow M / M_{1} \oplus M / M_{2}$, so is also an essential extension of $M$. Computation shows that the module $\left(M / M_{1} \oplus M / M_{2}\right) / M$ has length one and is a submodule of $S \oplus S$. Hence there is a nonsplit exact sequence

$$
0 \rightarrow M \rightarrow M / M_{1} \oplus M / M_{2} \rightarrow S \rightarrow 0
$$

or, $M$ has a decomposable proper simple extension.

Conversely let $M$ be as before and let $E$ be a proper simple extension of $M$ (such 
exist by (i)). Assume that $E$ is decomposable, say $E=L_{1} \oplus L_{2}$, where $L_{i} \neq 0$ $(i=1,2)$. Then $L_{i} \cap M \neq 0(i=1,2)$ since $E$ is an essential extension of $M$. Then

$$
E /\left(L_{1} \cap M\right) \oplus\left(L_{2} \cap M\right) \approx L_{1} / L_{1} \cap M \oplus L_{2} / L_{2} \cap M \approx S \oplus S
$$

where $S=E / M=L_{i}+M / M \approx L_{i} / L_{i} \cap M(i=1,2)$. But then $M /\left(L_{1} \cap M\right)+\left(L_{2} \cap M\right)$ is simple, and therefore $L_{1} \cap M \oplus L_{2} \cap M$ is maximal in $M$ but not a direct summand since $M$ is indecomposable.

Lemma 1.2 (SANDWICH Lemma). Let $A$ be an algebra over an algebraically closed field $K$, and let $E$ and $F$ be indecomposable left $A$-modules, situated such that $A_{1} \oplus A_{2} \subset E, F \subset B_{1} \oplus B_{2}$, where $0 \neq A_{i} \subset B_{i}$ are left $A$-modules, $B_{i} / A_{i}$ is simple $(i=1,2)$ and $B_{1} / A_{1} \approx B_{2} / A_{2}$. Then $E$ and $F$ are isomorphic.

Proof. If $H$ is a submodule of a direct sum $B_{1} \oplus B_{2}$, then as É. Goursat observed in 1889 [7] (see [14, p. 171]) there is an isomorphism

$$
\theta: \pi_{1}(H) / H \cap B_{1} \rightarrow \pi_{2}(H) / H \cap B_{2}
$$

where $\pi_{1}$ and $\pi_{2}$ are the projections and $\theta$ is defined as $\theta\left(b_{1}+H \cap B_{1}\right)=b_{2}+H \cap B_{2}$ where $b_{2}$ is any member of $B_{2}$ with $\left(b_{1}, b_{2}\right) \in H$. On the other hand, the submodule $H$ is completely determined by the data consisting of submodules $H_{i}^{\prime} \subseteq H_{i}$ of $B_{i}$. $(i=1,2)$ and an isomorphism $\theta: H_{1} / H_{1}^{\prime} \rightarrow H_{2} / H_{2}^{\prime}$ where $H$ is retrieved by the formula

$$
H=\left\{\left(b_{1}, b_{2}\right) \mid b_{1} \in H_{1}, b_{2}+H_{2}^{\prime}=\theta\left(b_{1}+H_{1}^{\prime}\right)\right\},
$$

where of course it holds that $H_{i}^{\prime}=H \cap B_{i}, H_{i}=\pi_{i}(H)(i=1,2)$. Now let $E$ and $F$ be indecomposable modules situated as above. Clearly $A_{i} \subseteq E \cap B_{i}$ and by indecomposability of $E$ we must have $\pi_{i}(E)=B_{i}$ and hence $E \cap B_{i}=A_{i}$. Similarly $F \cap B_{i}=A_{i}$ and $\pi_{i}(F)=B_{i}(i=1,2)$. Thus $E$ (resp. $F$ ) is determined completely by an isomorphism $\theta_{E}$ (resp. $\theta_{F}$ ) mapping $B_{1} / A_{1}$ onto $B_{2} / A_{2}$. But applying Schur's lemma we obtain $\xi \neq 0 \in K$ such that $\theta_{F}=\xi \theta_{E}$. Then it is easily checked that the automorphism $\xi^{-1} 1_{B_{1}} \oplus 1_{B_{2}}$ maps $E$ onto $F$.

The above lemma is in a sense dual to, and was inspired by Lemma C of [4], but we have removed the hypothesis of square-free socle which was required there.

We are now ready to prove the main result of this section.

THEOREM 1.3. Let $A$ be an algebra over an algebraically closed field $K$ such that every proper simple extension $E$ of a finitely generated stable indecomposable module $M$ has no repeated simple factor modules (i.e., E/NE contains at most one copy of any given simple module). Then $A$ has $\omega$-finite module type.

Proof. We prove by induction that for any positive integer $n$, there are only finitely many isomorphism classes of modules of length $n$. For $n=1$ this is clear since there are at most finitely many nonisomorphic simple modules. Assuming the statement for lengths less than $n$, let $E$ be an indecomposable module of length 
$n$. If $E$ has a decomposable maximal submodule $M_{1} \oplus M_{2}$, then the diagonal monomorphism $E \rightarrow E / M_{1} \oplus E / M_{2}$ yields the inclusions

$$
M_{1} \oplus M_{2} \subset E \subset E / M_{1} \oplus E / M_{2} .
$$

Then the Sandwich Lemma (1.2) shows that there is at most one choice for $E$ up to isomorphism, given the modules $M_{1}, M_{2}, E / M_{1}$, and $E / M_{2}$, all of which have smaller length. By induction, there are only finitely many choices for $E$. Now assume that $E$ is stable. If $E$ is injective, there are only finitely many choices for $E$, for then $E$ would be the injective envelope of a simple module which is determined up to isomorphism by its (simple) socle. So assume that $E$ is not injective, and let $M$ be any maximal submodule of $E$. Suppose $E / M=S$, say, where $S$ is simple, and let $E^{\prime}$ be another proper extension of $M$ by $S$. Then $E$ and $E^{\prime}$ are each essential extensions of $M$ and hence have isomorphic copies in $E(M)$, the injective envelope of $M$, and are of course isomorphic if these copies coincide in $E(M)$. Denote the copies also by $E$ and $E^{\prime}$, respectively, and assume that they are distinct submodules of $E(M)$. Let $E^{\prime \prime}=E+E^{\prime}$. Note that $E \cap E^{\prime}=M$, so that $\operatorname{Rad} E^{\prime \prime} \subseteq M$. Now $E^{\prime \prime}$ is a simple extension of $E$ (hence indecomposable by Proposition 1.1, (ii)) and by hypothesis, $E^{\prime \prime} / \operatorname{Rad} E^{\prime \prime}$ has no repeated simple factors. However,

$$
S \oplus S \approx E^{\prime \prime} \mid M \approx\left(E^{\prime \prime} / \operatorname{Rad} E^{\prime \prime}\right) /\left(M / \operatorname{Rad} E^{\prime \prime}\right)
$$

so that the completely reducible module $E^{\prime \prime} / \operatorname{Rad} E^{\prime \prime}$ contains a copy of $S \oplus S$. This contradiction shows that $E \approx E^{\prime}$, so that $E$ is uniquely determined by choice of $M$ and $S$, which have smaller lengths.

COROLlary 1.4. Let $A$ be an algebra satisfying the hypotheses of the above theorem. If in addition, $A$ has bounded module type, then $A$ has finite module type.

COROLlaRY 1.5 (CURTIS-JANS). Suppose $A$ is an algebra over an algebraically closed field $K$ such that every indecomposable finitely generated left A-module has square-free socle. Then $A$ has finite module type.

Proof. By $K$-duality it is equivalent to prove finite module type from the hypothesis that $M / N M$ is square-free for each finitely generated indecomposable module $M$ [4, p. 130]. The result then follows from the fact that such algebras already have bounded module type [4, dual of Lemma A].

Taking $K$-duals, Theorem 1.3 becomes

COROLlaRY 1.6. Let $A$ be an algebra over an algebraically closed field $K$ such that for each finitely generated costable indecomposable module $M$, the module $E$ appearing in any nonsplit exact sequence $0 \rightarrow S \rightarrow E \rightarrow M \rightarrow 0$ with $S$ simple has square-free socle. Then $A$ has w-finite module type.

We can now use the $K$-duality for algebras to further restrict the test class of indecomposables and strengthen these results. The next result is a sample application of this procedure. Recall an indecomposable module $E$ is stable-costable, if 
$E$ is both stable and costable. Then if $E$ is nonprojective as well as noninjective, the properties expressed in Proposition 1.1 hold for $E$ as well as their duals.

THEOREM 1.7. Let $A$ be an algebra over an algebraically closed field $K$ such that every finitely generated stable-costable indecomposable module $E$ has at least one of the following properties:

(i) Every proper simple extension $F$ of $E$ has $F / N F$ square-free.

(ii) Every proper simple coextension has square-free socle.

Then $A$ has w-finite module type.

Proof. The proof proceeds just as the proof of Theorem 1.3, except that now we may assume for the induction step that we have a stable-costable module $E$ of length $n$, using the dual of the Sandwich Lemma (or alternatively, applying the Sandwich Lemma for right modules to the right module $E^{*}=\operatorname{Hom}_{K}(E, K)$ ). Then application of either of (i) or (ii) completes the proof.

In the following theorem, $e$ denotes a primitive idempotent for $A$ and $B$ a right $A$-submodule of $e A$.

THEOREM 1.8. Let $A$ be an algebra over an algebraically closed field $K$ such that

(i) each right module eA/B has square-free socle,

(ii) each stable indecomposable left A-module has simple socle.

Then $A$ has w-finite module type.

Proof. As in the proof of Theorem 1.3 we need only show that there are at most finitely many nonisomorphic stable indecomposables. Since a stable indecomposable module $M$ has simple socle $S$, it is contained in the injective module $E(S)$. We show that the submodule lattice of $E(S)$ is finite. Assume on the contrary, that it is infinite. Then the $K$-dual $E(S)^{*}$ is of the form $e A$ for some primitive idempotent $e$ and the lattice of right submodules of $e A$ is also infinite, and of course is a modular lattice, so is nondistributive, and contains a projective root (cf. [10, p. 419], see also [1]) of right submodules $B_{i}(0 \leqq i \leqq 4)$ of $e A$. But then by properties of the

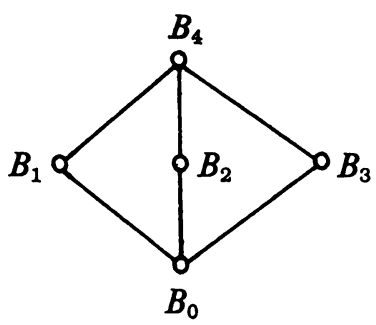

projective root (i.e., minimality of the $B_{i}$ over those below them in the diagram), $B_{1} / B_{0}$ and $B_{3} / B_{0}$ are isomorphic independent simple submodules of $e A / B_{0}$, contrary to the hypothesis (i). This completes the proof.

In [11], Janusz has constructed all the indecomposable modules for the group 
algebra of the finite group $\operatorname{LF}(2, p)$ over an algebraically closed field of characteristic $p$. Every indecomposable module has square-free socle and it is not too hard to check that the stable indecomposables over this algebra satisfy our hypothesis (ii). This group algebra has cyclic Sylow $p$-subgroups and has finite module type by Higman's theorem [9]. The above hypotheses are also satisfied by our matrix algebra example (nonquasifrobenius) below. In [12], Janusz shows that they are true for any group algebra of finite representation type.

Problem A. Let $\boldsymbol{A}$ be a finite-dimensional algebra of bounded module type over an algebraically closed field $K$. Assume also that $A$ is quasifrobenius. Is it true that every stable indecomposable module has simple socle?

Problem B. Describe the structure of the stable (costable, stable-costable) indecomposable modules for a group algebra.

Problem C. Characterize those algebras (over an arbitrary field) having the property that every stable indecomposable module has simple socle.

Remarks. 1. Tachikawa's results [16] show that every finitely generated indecomposable left module for the algebra $A$ consisting of all matrices of the form

$$
\left[\begin{array}{lll}
x & & \\
u & x & \\
v & w & y
\end{array}\right], \quad x, y, u, v, w \in K
$$

is quasiprimitive, i.e., is of the form $A e / L$ where $e$ is a primitive idempotent and $L$ is a left submodule of $A e$. Hence by Corollary 1.4 if $K$ is algebraically closed (or by direct computation of the endomorphism ring action of $A e_{x}=A\left(e_{11}+e_{22}\right)$ if $K$ is not algebraically closed) there are only finitely many indecomposables. The principal indecomposable $A e_{x}$ has nonsquare-free socle $K e_{31} \oplus K e_{32}$ where $e_{i j}$ is the $i j$ th matrix unit $(1 \leqq i, j \leqq 3)$.

2. C. W. Curtis and H. Bass (unpublished) have considered the question of whether the existence of a quasiprimitive module with nonsquare-free socle implies unbounded module type in general rings with minimum condition. The above example answers this question in the negative. See however, Theorem 3.1 to follow.

3. G. J. Janusz has informed me that his recent work [12] solves Problem A in the affirmative for group algebras. He has also solved Problem B in this setting. An indecomposable is stable (resp. costable, stable-costable) if and only if it has simple socle (resp, is quasiprimitive, is uniserial).

2. Quasifrobenius algebras with large kernels. The first result of this section was proved for finitely generated modules by C. W. Curtis and D. Zelinsky (unpublished) in a slightly different form. With their kind permission we include it here. Part of it appears in Heller's paper [8].

Proposition 2.1 (Heller, Curtis, Zelinsky). Let $A$ be a $Q F$ ring. Consider exact sequences of the form

$$
0 \rightarrow L \rightarrow P \rightarrow M \rightarrow 0
$$


where $P$ is simultaneously a projective cover of $M$ with kernel $L$ and an injective envelope of $L$ with quotient $M$. Then the following statements hold:

(i) Each nonprojective indecomposable module appears as $M$ in a sequence $\left(^{*}\right)$ with indecomposable $L$.

(ii) Each noninjective indecomposable module appears as $L$ in a sequence $\left(^{*}\right)$ with indecomposable $M$.

Proof. The statements (i) and (ii) are dual to each other and in view of the perfect duality over QF rings [5] it suffices to prove (i). Let $M$ be a nonprojective indecomposable module and $P(M)$ a projective cover [14, p. 93] of $M$ with kernel $L$. This leads to a commutative diagram

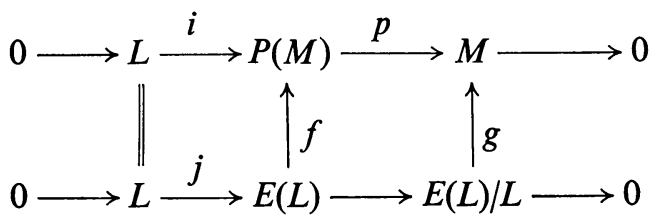

where the monomorphism $f$ exists by injectivity of $P(M)$ and $j$ is the injection of $L$ into $E(L)$. The map $g$ is filled in by the exactness of the rows and the commutativity of the left-hand square. Hence $P(M)=E(L) \oplus B$, say, and $M=P(L) / L=(E(L) / L)$ $\oplus B$, and as $M$ is indecomposable and not projective, $M=E(L) / L$. - Now if $L=$ $L_{1} \oplus L_{2}, L_{i} \neq 0(i=1,2)$, the exact sequence

$$
0 \rightarrow L=L_{1} \oplus L_{2} \rightarrow E(L)=E\left(L_{1}\right) \oplus E\left(L_{2}\right) \rightarrow M \rightarrow 0
$$

yields a decomposition of $M$ unless $L_{1}$ or $L_{2}$ is injective. If $L_{1}$ were injective, say, then $L$ would be a proper direct summand of $P(M)$, which would contradict the fact that $L_{1}$, being contained in the small (or superfluous) submodule $L$ of $P(M)$, is itself small in $P(M)$. We can conclude only that $L$ is indecomposable.

COROLlaRY 2.2. Let $M$ be any indecomposable module over a $Q F$ ring. Then $E(M) / M$ and the kernel of the projective cover of $M$ are indecomposable.

The following result appears to indicate that for finite module type in general QF rings with large kernels, it may not be necessary that each indecomposable module have square-free socle, but we have no explicit example to show this.

THEOREM 2.3. Let $A$ be a $Q F$ ring with large kernels having the property that if $M$ is an indecomposable finitely generated left $A$-module, then the socle of $M$ is cyclic as a right module over the endomorphism ring $R=\operatorname{End}_{A}(M)$. Then $A$ has $\omega$-finite module type.

Proof. Let $n$ be as in the proof of Theorem 1.3. For the induction step take $E$ stable indecomposable. If $M$ is a maximal submodule of $E$, then $M_{0}=E(M) / M$ is indecomposable by Corollary 2.2 and $E / M$ is a simple submodule. Now if $E^{\prime}$ 
is indecomposable containing $M$ with $E^{\prime} / M \approx E / M$, there is by hypothesis a submodule $E^{\prime \prime} / M$ of $M_{0}$ equipped with endomorphisms (hence automorphisms by the hypothesis of large kernels) $\varphi, \varphi^{\prime}$ of $M_{0}$ with $\varphi\left(E^{\prime \prime} \mid M\right)=E / M, \varphi^{\prime}\left(E^{\prime \prime} \mid M\right)=E^{\prime} \mid M$. But then $\varphi$ and $\varphi^{\prime}$ can be lifted to endomorphisms $\Phi, \Phi^{\prime}$ of $E(M)$, since $E(M)$ is projective, satisfying $\Phi\left(E^{\prime \prime}\right)=E, \Phi^{\prime}\left(E^{\prime \prime}\right)=E^{\prime}$. To see that these last two equalities hold, note that the Fitting decomposition for $E(M)$ with respect to $\Phi$ is $E(M)$ $=\bigcap \Phi^{n}(E(M)) \oplus \bigcup \operatorname{Ker} \Phi^{n}$, and since $\varphi$ is an automorphism of $E(M) / M$ we have $\bigcup \operatorname{Ker} \Phi^{n} \subseteq M$, and hence the decomposition $M=\bigcup \operatorname{Ker} \Phi^{n} \oplus M \cap$ $\left[\cap \Phi^{n}(E(M))\right]$. Since $M$ is indecomposable, either $\bigcup \operatorname{Ker} \Phi^{n}=0$ or $M \cap$ $\left[\cap \Phi^{n}(E(M))\right]=0$, hence $\bigcap \Phi^{n}(E(M))=0$ since $M$ is essential in $E(M)$. In the latter case, $\Phi$ would be nilpotent, an impossibility since $\varphi$ is an automorphism. By commutativity of the lifting diagram we have at least $\Phi\left(E^{\prime \prime}\right)+M=E$ and since $\Phi$ preserves the length of $E^{\prime \prime}, E / \Phi\left(E^{\prime \prime}\right) \approx M / M \cap \Phi\left(E^{\prime \prime}\right)=0$ so that $M \subseteq \Phi\left(E^{\prime \prime}\right)$ and the desired equality $\Phi\left(E^{\prime \prime}\right)=E$ holds. Similarly $\Phi^{\prime}\left(E^{\prime \prime}\right)=E^{\prime}$ holds. Hence $E \approx E^{\prime}$ and $E$ is determined, once $M$ and $E / M$ are known, and these are modules of smaller length.

The next result will show, however, that cyclicity of the socle, and hence cyclicity of the homogeneous components of the socle of $M$ over the endomorphism ring $R$ of $M$ actually implies square-free socle in case the base field is algebraically closed.

Proposition 2.4. Let $A$ be an algebra over an algebraically closed field and let $M$ be an indecomposable module of finite length having large kernels. Then any endomorphism of $M$ has scalar action on the socle of $M$.

Proof. Since the base field is algebraically closed, any endomorphism of $M$ can be written as scalar plus nilpotent, and our hypothesis guarantees that the kernel of any nilpotent endomorphism contains the socle.

Remarks. 1. This shows that the left $A$-socle is contained in the right $R$-socle of $M$ when $M$ has large kernels. We do not know if the opposite inclusion holds for QF rings, but this should not be a difficult question. However, in general, neither inclusion holds - see the example in Remark 1 at the end of $\S 1$. The right $e_{x} A e_{x}$-socle of the indecomposable module $A e_{x}\left(e_{x}=e_{11}+e_{22}\right)$ of the matrix algebra there is $\mathrm{Ke}_{21} \oplus \mathrm{Ke}_{32}$. Note that in the setting of Proposition 2.4, cyclicity of the homogeneous components of the socle of $M$ over $R$ is equivalent to their irreducibility over $R$ in view of that result. In the matrix algebra example, it is interesting to note that the intersection of the left $A$ - and right $e_{x} A e_{x}$-socles is irreducible on either side.

2. Note that the hypothesis of square-free socle implies large kernels-if $M$ is indecomposable of finite length and $R=\operatorname{End}_{A}(M), \mathscr{N}=\operatorname{Rad} R$, then for $S$ a simple submodule of $M$ we have $S=S R \supset S \mathscr{N}$ and since $S \neq S \mathscr{N}, S \mathscr{N}=0$ as a left submodule of $S$, assuming square-free socle. We doubt if the converse is true, but the results to follow give some information in this direction. 
THEOREM 2.5. Let $A$ be a $Q F$ algebra with large kernels over an algebraically closed field $K$, and let $M$ be an indecomposable direct summand of a finitely generated module $L$ such that $L$ is carried into itself by every automorphism of its injective envelope $E(L)$. Then either $E(M) / M$ has square-free socle, or $A$ has infinitely many nonisomorphic modules of the same composition length.

Proof. First assume that $L$ is itself indecomposable, so $M=L$. If $M$ is injective, there is no problem, but otherwise $B=E(M) / M$ is indecomposable by Corollary 2.2. Also by Proposition 2.4 the automorphisms of $B$ have scalar action on the left $A$-socle of $B$. Let $S$ be a simple left $A$-module and suppose that $E_{1} / M$ and $E_{2} / M$ are copies of $S$ in $B$, say $A x=E_{1} / M$ and $A y=E_{2} / M$, such that there is an isomorphism $\varphi: E_{1} \rightarrow E_{2}$. Now $\varphi$ can be extended to an automorphism $\Phi$ of $E(M)$ and since $M$ is held invariant, $\Phi$ induces an automorphism of $B$ taking $A x$ into $A y$, but since the action is scalar we must have $A x=A y$. Hence we must conclude that $E_{1}=E_{2}$ as submodules of $E(M)$ containing $M$. This shows that if the socle of $B$ is not square-free, there are such simple submodules $A x \neq A y$, and the resulting infinite collection of simple submodules of $B$ of the form $A(x+\theta y)(\theta \in K)$ gives rise to infinitely many nonisomorphic simple extensions of $M$, all having the same composition length.

Now let $L$ be an arbitrary finitely generated module which is held invariant by automorphisms of its injective envelope. Let $E / L$ be an indecomposable direct summand of $E(L) / L$, and assume that its socle is not square-free, say $S_{1} \oplus S_{2}$ $\subset E / L$, with $S_{1} \approx S_{2}, S_{i}=E_{i} / L(i=1,2)$. Now if $E_{1} \approx E_{2}$ by $\varphi: E_{1} \rightarrow E_{2}$, then $\varphi$ has an extension $\Phi_{0}$ to all of $E(L)$ since $E(L)$ is injective, which induces an endomorphism $\Phi$ of $E(L) / L$. Thus $\Phi$ can be considered as a matrix $\left(\varphi_{i j}\right)$ of homomorphisms between the various indecomposable summands in a finite decomposition of $E(L) / L$ in which $E / L$ appears, and we may assume that $\varphi_{11}$ comes from $\operatorname{End}_{A}(E / L)$. Since $\Phi\left(S_{1}\right)=S_{2}$ the same holds for $\varphi_{11}$, but as $E / L$ is indecomposable, $\varphi_{11}$ has scalar action of the socle of $E / L$, and we have $S_{1}=S_{2}$, a contradiction. Hence $E_{1}$ and $E_{2}$ are nonisomorphic and the argument in the previous case shows that there are infinitely many nonisomorphic modules of the same length.

Now if $L=L_{1} \oplus \cdots \oplus L_{n}$ is any decomposition of $L$ into indecomposable modules, we have $E(L) / L=E\left(L_{1}\right) / L_{1} \oplus \cdots \oplus E\left(L_{n}\right) / L_{n}$ with all the summands indecomposable by Corollary 2.2. To complete the proof, choose $M$ to be any indecomposable direct summand of $L$ and use the Krull-Schmidt theorem to conclude that $E(M) / M$ has square-free socle if $A$ has finite module type.

COROLlaRy 2.6. Let $A$ be a $Q F$ algebra with large kernels over an algebraically closed field having $\omega$-finite module type. Then any indecomposable injective module has a finite lattice of submodules, and contains only one copy of each submodule.

Proof. We first show that each submodule of $E(S)$ is carried into itself by all automorphisms of $E(S)$, where $S$ is simple. This is clearly true for the unique submodule $S$ of length one. Let $M$ be a submodule of smallest length which is not. 
Let $\varphi$ be an automorphism with $\varphi(M) \neq M$. By induction, a maximal submodule $M_{1}$ is held invariant by any automorphism of $E(S)$ so that $\varphi(M)+M / M_{1}$ is of the form $S^{\prime} \oplus S^{\prime}$ for some simple $S^{\prime}$. Since the module $M_{1}$ has $E(S)$ for its injective envelope, Theorem 2.5 applies to yield a contradiction. Our conclusion is equivalent to the second statement of the corollary since any isomorphism between submodules of $E(S)$ extends to an automorphism of $E(S)$. Now assume that the submodule lattice of $E(S)$ is infinite. Since it is modular, there is a projective root consisting of submodules $B_{i}(0 \leqq i \leqq 4)$ (as in the proof of Theorem 1.8) with $B_{1} / B_{0}$ and $B_{3} / B_{0}$ isomorphic independent simple submodules. Then by the first part and another application of Theorem 2.5 we are through.

Corollary 2.7. Let $A$ be as in Theorem 2.5 and suppose that each finitely generated indecomposable module $M$ has the property described there. Suppose also that $A$ has $\omega$-finite module type. Then $A$ has finite module type.

Proof. Any indecomposable has the form $E(M) / M$ by Corollary 2.2 and application of Theorem 2.5 shows that any finitely generated indecomposable has square-free socle, so we are through by the theorem of Curtis and Jans (Corollary 1.5).

3. Relations between bounded and finite module type. In this section we combine the results of the preceding sections to gain some additional information on the general question of when bounded module type implies finite module type. The first result is a generalization of a standard procedure for constructing large indecomposables by interlacing methods. (see [3], [17], [2]) and in its present form is really a result on abelian categories, by the full imbedding theorem [6, p. 101].

THEOREM 3.1. Let $A$ be a ring with unit. Let $M$ be an indecomposable left $A$ module having finite composition length. Let $R=\operatorname{End}_{A}(M)$ and suppose that $C$ and $C^{\prime}$ are isomorphic left $A$-submodules of $M$ with $C R \cap C^{\prime} R=0=C \mathscr{N}=C^{\prime} \mathscr{N}$ and such that $\operatorname{Ext}_{A}{ }^{1}(M, C)=0$. Then $A$ has indecomposable left modules of arbitrary large ( finite) composition length.

Proof. First assume that $C$ is cyclic. Let $\varepsilon_{i}: M \rightarrow M_{i}$ be an isomorphism $(1 \leqq i \leqq n)$ and let $\rho: C \rightarrow C^{\prime}$ be an isomorphism of left $A$-modules. Choose $x \in C$ a generator and let $y=\rho(x) \in C^{\prime}$. Then we have $A x+x R \cap A y+y R=0$. Let $M^{n}=M_{1} \oplus \cdot, \oplus M_{n}$. Let $L$ be the left $A$-submodule of $M^{n}$ generated by the elements

$$
y \varepsilon_{1}-x \varepsilon_{2}, y \varepsilon_{2}-x \varepsilon_{3}, \ldots, y \varepsilon_{n-1}-x \varepsilon_{n}
$$

where we have identified $M_{i}$ with its copy in $M^{n}(1 \leqq i \leqq n)$. Let $M^{(n)}=M^{n} / L$. We shall prove that $M^{(n)}$ is indecomposable as a left $A$-module. Its length may be chosen as large as desired, for the length of $L$ is at most $n-1$ times the length of $A x$ and the length of $M^{n}$ is at least $2 n$ times the length of $A x$. In order to show that $M^{(n)}$ is indecomposable, it suffices to show that any endomorphism is either 
nilpotent or invertible, since $M^{(n)}$ has finite length. Let $\varphi$ be an endomorphism of $M^{(n)}$. We want to lift $\varphi$ to an endomorphism $\Phi$ of $M^{n}$ which makes the following diagram commutative.

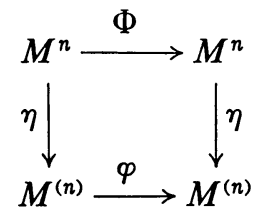

where the vertical maps $\eta$ are the natural epimorphism. It suffices to lift $\varphi \eta$. But the exact sequence

$$
0 \rightarrow L \rightarrow M^{n} \rightarrow M^{(n)} \rightarrow 0
$$

yields the exact sequence

$$
\operatorname{Hom}_{A}\left(M^{n}, M^{n}\right) \rightarrow \operatorname{Hom}_{A}\left(M^{n}, M^{(n)}\right) \rightarrow \operatorname{Ext}_{A}{ }^{1}\left(M^{n}, L\right) .
$$

Now $L$ is a direct sum of $n-1$ copies of $A x$, and since Ext commutes with finite direct sums in each variable, our hypothesis guarantees that the right-hand term is zero, and this insures that the lifting $\Phi$ is possible. Now $\Phi$ can be considered as an $n \times n$ matrix $\Phi=\sum_{i, j} \epsilon_{i j} \alpha_{i j}$ where $\epsilon_{i j}$ is the $i j$ th matrix unit and $\alpha_{i j} \in R=\operatorname{End}_{A}(M)$, where the right action is for $m \in M$,

$$
m \varepsilon_{k} \epsilon_{i j} \alpha_{i j}=\sum_{i} \delta_{i k}(m) \alpha_{i j} \varepsilon_{j}=m \alpha_{k j} \varepsilon_{j} .
$$

Since $\Phi$ must hold $L$ invariant, we have for $1 \leqq s \leqq n-1$

which must also be of the form

$$
\left(y \varepsilon_{s}-x \varepsilon_{s+1}\right) \Phi=\sum_{j}\left(y \alpha_{s, j}-x \alpha_{s+1, j}\right)
$$

$$
a_{s, 1} y \varepsilon_{1}+\sum_{j=2}^{n-1}\left(a_{s, j} y-a_{s, j-1} x\right) \varepsilon_{j}-a_{s, n-1} x \varepsilon_{n}
$$

where $a_{s, j} \in A(1 \leqq j \leqq n-1)$. This yields the equations

$$
\begin{aligned}
y \alpha_{s, 1}-x \alpha_{s+1,1} & =a_{s, 1} y, \\
y \alpha_{s, j}-x \alpha_{s+1, j} & =a_{s, j} y-a_{s, j-1} x, \quad 2 \leqq j \leqq n-1, \\
y \alpha_{s, n}-x \alpha_{s+1, n} & =-a_{s, n-1} x .
\end{aligned}
$$

Since each element of $R$ is either nilpotent or invertible, it is readily determined from these equations that the elements off the diagonal of the matrix $\left(\alpha_{i j}\right)$ are all nilpotent, and those on the diagonal are either all invertible or all nilpotent together. This shows that $\left(\alpha_{i j}\right)$ is either nilpotent or invertible and the same holds for $\varphi$ by the commutativity of the diagram.

In the general case, $L$ is taken to be the direct sum of the $n-1$ copies of $C$ of the form

$$
L_{s}=\left\{\rho(x) \varepsilon_{s}-x \varepsilon_{s+1} \mid x \in C\right\} \quad(1 \leqq s \leqq n-1)
$$

and the above equations still apply with $y=\rho(x)$ as $x$ varies through $C$. 
RemarK. The hypothesis Ext $(M, C)=0$ can be replaced by the hypothesis that $M^{n} \rightarrow M^{n} / L \rightarrow 0$ exact implies that $\operatorname{Hom}_{A}\left(M^{n}, M^{n}\right) \rightarrow \operatorname{Hom}_{A}\left(M^{n}, M^{n} / L\right) \rightarrow 0$ is exact, which is weaker. Also note that only those endomorphisms of $M^{n}$ which take $L$ into $L$ need be lifted.

TheOREM 3.2 (JANS, TACHIKAWA, ColBy). Let $A$ be a ring with minimum condition on left ideals having an infinite two-sided ideal lattice. Then $A$ has unbounded module type.

Proof. As in the proof of Theorem 1.8 find a projective root consisting of twosided ideals $B_{i}(0 \leqq i \leqq 4)$ with now $B_{1} / B_{0}$ and $B_{3} / B_{0}$ isomorphic independent sub$(A, A)$-bimodules. Then for some primitive idempotent $e$, choose nonzero elements $x \in B_{1} e / B_{0} e, y \in B_{3} e / B_{0} e$ such that $A x \approx A y$ as simple left $A$-modules. Then $M=$ $A e / B_{0} e$ is indecomposable, and $B_{0} e$ is invariant under the action of the endomorphisms of $M$, which are all induced by right multiplications by elements of $e A e$. It follows that $\left(B_{0} e\right)^{n}$ is invariant under the endomorphisms of $(A e)^{n}$. Thus following the proof of Theorem 3.1, any endomorphism of $M^{(n)}=M^{n} / L$ can be lifted first to one of $(A e)^{n}$ by projectivity of $(A e)^{n}$, and then dropped to one of $M^{n}$ so that the required diagram commutes.

For our purposes we need the following version of Theorem 3.1.

Corollary 3.3. Let $A$ be a $Q F$ algebra over an algebraically closed field $K$ and assume that $A$ has bounded module type. Then if $M$ is any indecomposable finitely generated left A-module with large kernels, the $S$-part of the socle of $M$ is square-free, provided $\operatorname{Ext}_{A}{ }^{1}(M, S)=0$.

Proof. By Proposition 2.4, the automorphisms of $M$ have scalar action on the socle, so if $R=\operatorname{End}_{A}(M)$ then for $x$ in the socle, $A x+x R=A x+K x=A x$, and similarly for $A y$. This completes the proof.

THEOREM 3.4. Let $A$ be a $Q F$ algebra with large kernels over an algebraically closed field. Suppose that $A$ has bounded module type and that each stable indecomposable finitely generated module $E$ has a maximal submodule $M$ such that $\operatorname{Hom}_{A}(M, E / M)=0$. Then $A$ has finite module type.

Proof. We utilize the proof of Theorem 1.3 to reduce to the stable case so we may assume that $E$ is a stable indecomposable of finite length. Let $M$ be a maximal submodule guaranteed by our hypothesis and let $S=E / M$. If we show that the $S$-part of the socle of $E(M) / M$ is square-free we will be through as in the proof of Theorem 1.3 for there will be room for only one extension of $M$ by $S$ in $E(M)$, and $M$ and $S$ are of smaller length. But the exact sequence

$$
0 \rightarrow M \rightarrow E(M) \rightarrow E(M) / M \rightarrow 0
$$

yields the exact sequence

$$
\operatorname{Hom}_{A}(M, S) \rightarrow \operatorname{Ext}_{A}^{1}(E(M) / M, S) \rightarrow \operatorname{Ext}_{A}^{1}(E(M), S)
$$


where the first term is zero by hypothesis and the last term is zero since $E(M)$ is projective. An application of Corollary 3.3 concludes the proof.

THEOREM 3.5. Let $A$ be a $Q F$ algebra with large kernels over an algebraically closed field. Suppose that $A$ has bounded module type and that each finitely generated costable indecomposable module $E$ has a factor module $E / S$ with $\operatorname{Hom}_{A}(S, E / S)=0$, where $S$ is simple. Then $A$ has finite module type.

Proof. This result follows from Theorem 3.4 by $K$-duality, where $K$ is the base field.

Finally, combining these duality results we may restrict ourselves still further, as in Theorem 1.7 to stable-costable modules, and we have the following result.

THEOREM 3.6. Let $A$ be a $Q F$ algebra with large kernels over an algebraically closed field having bounded module type and assume that each finitely generated stable-costable indecomposable module $E$ satisfies at least one of the following dual conditions:

(i) $E$ has a maximal submodule $M$ with $\operatorname{Hom}_{A}(M, E / M)=0$,

(ii) $E$ has a factor module $E / S$ where $S$ is simple and $\operatorname{Hom}_{A}(S, E / S)=0$. Then $A$ has finite module type.

As an application, we make a contribution to an open question mentioned in the paper of Curtis and Jans [4] which asks whether the hypothesis that for any indecomposable $M$, either the socle $s(M)$ or the top, $M / N M$ is square-free implies finite module type. Kent R. Fuller has pointed out to me that the condition (ii) of the following result holds for indecomposable generalized uniserial rings which are not already uniserial, by a result of Kupisch [13]. This condition also holds for the group algebra over $\operatorname{LF}(2, p)$ in [11].

COROLlARY 3.7. Let $A$ be a $Q F$ algebra with large kernels over an algebraically closed field having the properties that

(i) for each finitely generated indecomposable module $M$, either $s(M)$ or $M / N M$ is square-free,

(ii) any indecomposable module of length two has distinct composition factors. Then $A$ has finite module type.

Proof. First, $A$ has bounded module type since any indecomposable module is either contained in the minimal completely faithful injective module (see [4, Lemma A]) or is cyclic. Let $E$ be a stable-costable indecomposable module which is finitely generated. If $E / N E$ is square-free then condition (i) of Theorem 3.6 is satisfied, and if $s(E)$ is square-free then (ii) is satisfied. Hence $A$ has finite module type.

\section{REFERENCES}

1. G. Birkhoff, Lattice theory, Amer. Math. Soc. Colloq. Publ., Vol. 25, Amer. Math. Soc., Providence, R. I., 1948; rev. ed. 1966.

2. H. Brummund, Über Gruppenringe mit einem Koeffizientenkörper der characteristic p, Inaugural Dissertation, Munster, 1939. 
3. R. Colby, On indecomposable modules over rings with minimum condition, Pacific J. Math. 19 (1966), 23-33.

4. C. W. Curtis and J. P. Jans, On algebras with a finite number of indecomposable modules, Trans. Amer. Math. Soc. 114 (1965), 122-132.

5. J. Dieudonné, Remarks on quasi-Frobenius rings, Illinois J. Math. 2 (1958), 346-354.

6. P. Freyd, Abelian categories, Harper and Row, New York, 1964.

7. E. Goursat, Sur les substitutiones orthogonales ..., Ann. Sci. École Norm. Sup. (3) 6 (1889), 9-102.

8. A. Heller, Indecomposable representations and the loop-space operation, Proc. Amer. Math. Soc. 12 (1961), 640-643.

9. D. G. Higman, Indecomposable representations at characteristic p, Duke Math. J. 21 (1954), 377-381.

10. J. P. Jans, On the indecomposable representations of algebras, Ann. of Math. (2) 66 (1957), 418-429.

11. G. J. Janusz, Indecomposable representations of groups with a cyclic Sylow subgroup, Trans. Amer. Math. Soc. 125 (1966), 288-295.

12. - Indecomposable modules for finite groups, Trans. Amer. Math. Soc. (to appear).

13. H. Kupisch, Beitrage zur Theorie nichthalbeinfachen Ringe mit Minimalbendingung, J. Reine Angew. Math. 201 (1959), 100-112.

14. J. Lambek, Lectures on rings and modules, Blaisdell, Waltham, Mass., 1966.

15. K. Morita, Duality for modules and its application to the theory of rings with minimum condition, Sci. Rep. Tokyo Kyoiku Daigaku Sect. A 6 (1958), 38-142.

16. H. Tachikawa, On rings for which every indecomposable right module has a unique maximal submodule, Math. Z. 71 (1959), 200-222.

17. - A note on algebras of unbounded representation type, Proc. Japan Acad. 36 (1960), 59-61.

18. Y. Utumi, On a theorem on modular lattices, Proc. Japan Acad. 35 (1959), 16-21.

UNIVERSITY OF OREGON, EUGENE, OREGoN

UNIVERSITY OF NEBRASKa, Lincoln, Nebraska 